\title{
Caracterización de la Impactación de Segundos Molares Mandibulares Permanentes en una Población Sudamericana
}

\author{
Characterization of Permanent Mandibular Second Molars \\ Impaction in a Southamerican Population
}

\author{
Diana Ramírez Ossa'; Juan Carlos Upegui Zea²; Gabriel Emilio Espinal Botero³ \& Rosa Cristina Chaurra Marín ${ }^{4}$
}

RAMÍREZ, O. D.; UPEGUI, Z. J. C.; ESPINAL, B. G. E. \& CHAURRA, M. R. C. Caracterización de la impactación de segundos molares mandibulares permanentes en una población sudamericana. Int. J. Odontostomat., 11(2):165-172, 2017.

RESUMEN: El objetivo de este estudio fue describir las características epidemiológicas de segundos molares mandibulares permanentes impactados unilateralmente en una muestra de la ciudad de Medellín, Colombia. Se trató de un estudio retrospectivo en el cual se evaluaron radiografías panorámicas de 6785 pacientes, de las cuales se analizaron un total de 28 radiografías en la muestra final. Fueron correlacionados el segundo molar mandibular impactado (grupo de estudio) y el contralateral no impactado (grupo control) con respecto a tres ángulos de referencia (intermolar, del plano oclusal e interplanar), el sexo y lado de impactación. Se encontró una prevalencia de 3,5 por cada 1000 radiografías evaluadas. El lado de mayor impactación fue el derecho; dos de los tres ángulos analizados (intermolar e interplanar) fueron los que arrojaron las diferencias más grandes entre el grupo de estudio y el grupo control. No se encontraron diferencias estadísticamente significativas según el sexo. Se concluyó que la prevalencia de segundos molares mandibulares permanentes impactados es baja, siendo mayor en el lado derecho, sin diferencias por sexo, y de preferencia el uso del ángulo interplanar como medida para la evaluación del riesgo de impactación $\left(>7,9^{\circ}\right)$.

PALABRAS CLAVE: dientes impactados, molar, radiografía panorámica, epidemiología.

\section{INTRODUCCIÓN}

La impactación es el fracaso en la erupción de un diente (Suri et al., 2004), causada por la posición ectópica del germen dentario y obstáculos en el camino de emergencia a la cavidad oral (Shokeir, 1974; Evans, 1988; Varpio \& Wellfelt, 1988; Raghoebar et al., 1991; Andreasen et al., 1997; Vedtofte et al., 1999; Suri et al.; Frazier-Bowers et al., 2010). En la dentición permanente se presenta con mayor frecuencia en los terceros molares, seguidos de los caninos y premolares (Fardi et al., 2011).
La prevalencia de impactación de segundos molares mandibulares es relativamente baja, con reportes que oscilan entre el $0,15 \%$ al $1,4 \%$ (Varpio \& Wellfelt; Bondemark \& Tsiopa, 2007; Shapira et al., 2011; Fu et al., 2012; Cassetta et al., 2013), pero encontrándose reportes mayores según el tipo étnico $(2,3$ $\%$ en chino-americanos) (Shapira et al.). Ocurre generalmente en el arco mandibular, de forma unilateral y en pacientes de ambos sexos (Varpio \& Wellfelt). La causa más frecuente de impactación de segundos

\footnotetext{
${ }^{1}$ Odontóloga, Especialista Clínico en Odontología Integral del Adolescente y Ortodoncia, Docente Ocasional, Facultad de Odontología, Universidad de Antioquia, Medellín, Colombia.

${ }^{2}$ Odontólogo, Especialista en Odontología Integral del Niño y Ortopedia Maxilar, Especialista en Ortodoncia, Docente Asociado, Facultad de Odontología, Universidad de Antioquia, Medellín, Colombia.

${ }^{3}$ Odontólogo, Especialista Clínico en Odontología Integral del Niño y Ortopedia Maxilar, Docente Asistente, Facultad de Odontología, Universidad de Antioquia, Medellín, Colombia.

${ }^{4}$ Odontóloga, estudiante de la Especialización Clínica en Ortodoncia. Facultad de Odontología, Universidad de Antioquia, Medellín, Colombia.

* Artículo derivado de la investigación realizada como requisito para optar al título de Especialista Clínico en Ortodoncia de uno de los coautores, apoyado por el Grupo de Investigación en Odontología del Niño y Ortodoncia - GIONORTO de la Facultad de Odontología de la Universidad de Antioquia.
} 
molares se relaciona con el apiñamiento posterior, que genera una inclinación de su eje longitudinal hacia mesial (Evans; Varpio \& Wellfelt; Wellfelt \& Varpio, 1988; Shapira et al.).

El diagnóstico de un segundo molar impactado es importante para evitar la aparición de lesiones de caries en la zona afectada, daños en la raíz distal del primer molar, problemas periodontales, endodónticos y oclusales (Raghoebar et al.; Kurol, 2006; Miyahira et al., 2008; Magnusson \& Kjellberg, 2009). Adicionalmente, las intervenciones ortodóncico-quirúrgicas para su tratamiento son complejas, incómodas y de altos costos para el paciente (Power \& Short, 1993; Shapira et al.). El momento ideal para el tratamiento es entre los 11 y 14 años, etapa en que aún el desarrollo de las raíces del segundo molar permanente no ha concluido (Wellfelt \& Varpio; Kokich \& Mathews, 1993) y los terceros molares están en los primeros estadios de formación (Shapira et al., 1998). La detección temprana puede realizarse por medio de la aplicación de análisis angulares medidos en radiografías panorámicas (Evans; Cho et al., 2008; Shapira et al.; Sonis \& Ackerman, 2011; Fu et al.; Cassetta et al.), las cuales, además de ser un método diagnóstico con bajo nivel de radiación y común en la consulta odontológica, proveen información valiosa sobre dientes no erupcionados (Lindauer et al., 1992; Warford et al., 2003). Se ha confirmado mediante cálculos matemáticos y pruebas experimentales, que las imágenes de este tipo de radiografías son confiables para realizar medidas angulares con mucha precisión (Frykholm et al., 1977).

En la literatura, no se encuentra información epidemiológica de esta entidad clínica en poblaciones latinas que permitan hacer comparaciones con otras etnias a nivel mundial, por tanto, el objetivo de este estudio es describir las características epidemiológicas de segundos molares mandibulares permanentes impactados unilateralmente en una muestra de Medellín, Colombia.

\section{MATERIAL Y MÉTODO}

Diseño y fuentes de información. Estudio retrospectivo cuya población de estudio fueron radiografías panorámicas de pacientes provenientes de la Facultad de Odontología de la Universidad de Antioquia y de diferentes centros de práctica privada de ortodoncistas de la ciudad de Medellín, Colombia.
La muestra final se definió teniendo en cuenta los siguientes criterios de inclusión: (1) radiografías de pacientes mayores de 11 años con diagnóstico de segundo molar mandibular impactado unilateralmente, definido como diente no erupcionado aun cuando tiene más de $2 / 3$ de formación radicular o presenta estadio de Nolla 8 o superior, y cuya cúspide mesial está en íntimo contacto con el primer molar a nivel del tercio cervical o apicalmente (Shapira et al.) (Fig. 1); (2) calidad de la radiografía (contraste y detallado de la imagen); (3) presencia e integridad del primer molar mandibular permanente; (4) pacientes con dentición permanente en el arco mandibular; (5) sin ausencias congénitas o supernumerarios que comprometieran el área clínica de la investigación, ni tratamiento de ortodoncia o presencia de distalizadores en el arco inferior. Como criterios de exclusión se consideraron (1) la presencia del segundo molar mandibular a nivel de la rama, lesiones periapicales en el área de estudio, tumores óseos u odontogénicos, y (2) pacientes con síndromes o enfermedades sistémicas.

La muestra se realizó por conveniencia, dado que los porcentajes de incidencia de esta entidad son muy bajos. El trabajo de campo se realizó entre julio de 2014 y diciembre de 2015 (Fig. 2), por medio de la valoración de radiografías panorámicas tanto digitales como convencionales de 6785 pacientes, con un total 40 pacientes con la condición objeto de estudio. Tras aplicar los criterios de inclusión y exclusión, se descartaron 12 pacientes, 4 por presentar la condición bilateral, 5 con dentición mixta y 3 con aparatología ortopédica en boca, para una muestra final de 28 pacientes de ambos sexos con la condición de segundos molares mandibulares impactados unilateralmente, con un rango de edad entre los 11 y 25 años.

Las radiografías convencionales fueron digitalizadas con un scanner de doble pantalla $\left(\mathrm{HP}^{\circledR}\right.$ Scanjet 64050), y las digitales fueron almacenadas como formato JPG. Con la ayuda del programa Solid Edge ST18 de Siemens ${ }^{\odot}$, se realizó el trazado de las variables angulares del estudio, las cuales son confiables ya que los estudios soportan que el rango de error para este tipo de medidas es muy poco, aún entre panorámicas tomadas en diferentes equipos (Frykholm et al.; Stramotas et al., 2000).

Para garantizar la reproducibilidad de las lecturas de los ángulos, se realizó una evaluación de variabilidad intrasujeto y se utilizó el coeficiente de correlación intraclase $(\mathrm{CCl})$, obteniendo valores superiores al $80 \%$ en todas las mediciones (Tabla I). 


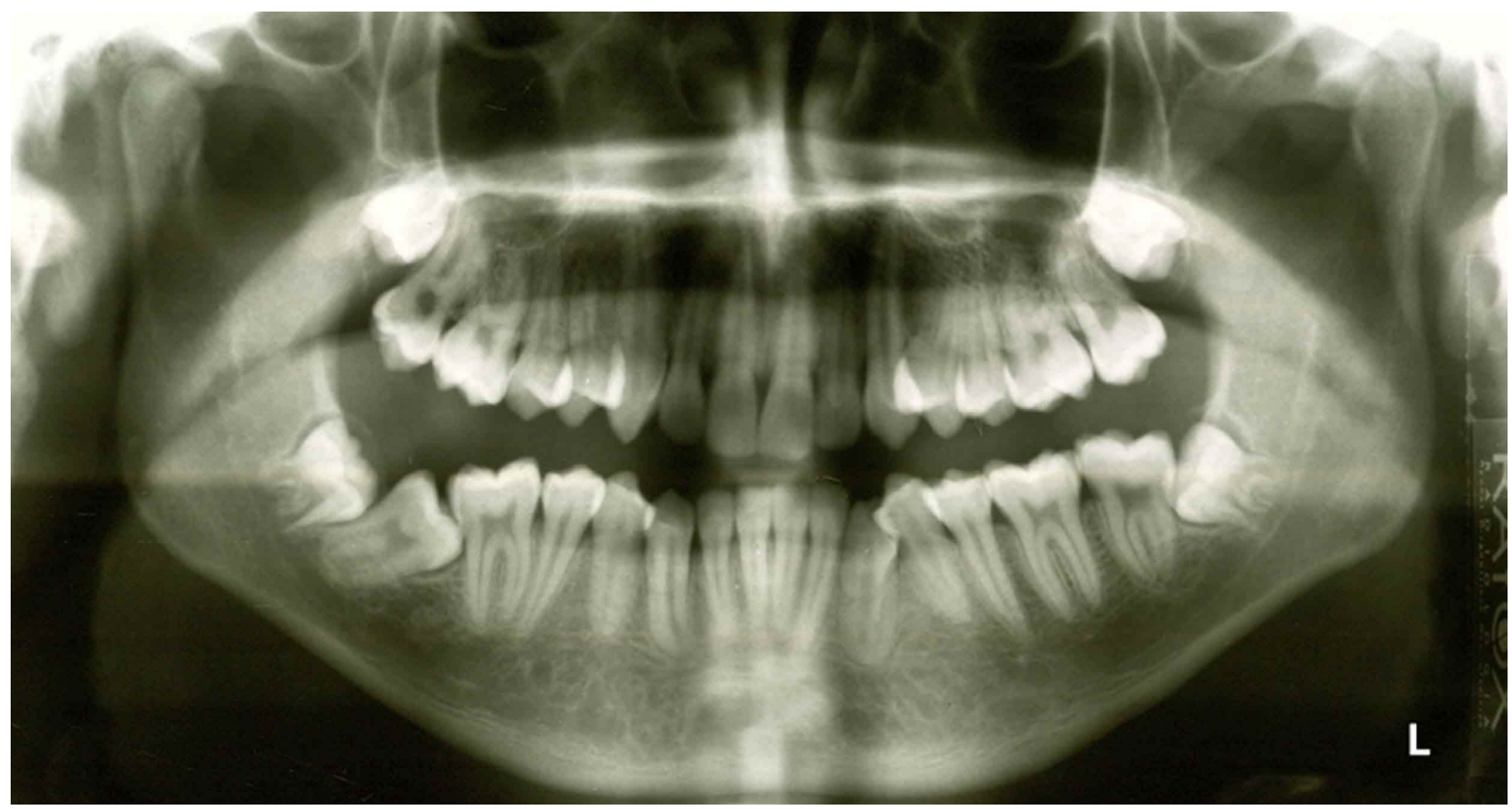

Fig. 1. Segundo molar mandibular impactado.

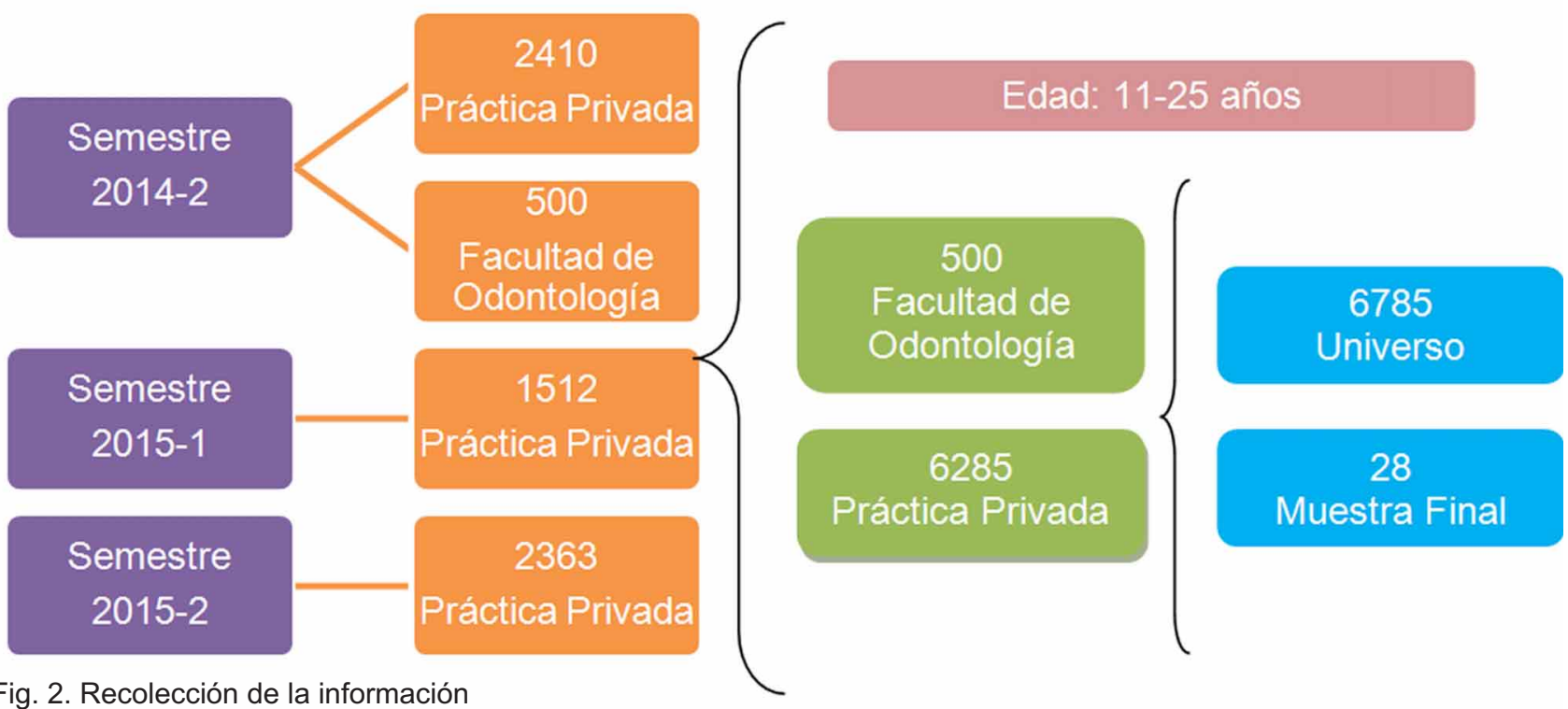

Fig. 2. Recolección de la información

Variables: Se analizó la relación de segundos molares mandibulares impactados y no impactados con respecto a otros planos de referencia, clasificados según edad, sexo y lado de impactación. Se midieron tres ángulos reportados en la literatura para predecir el riesgo de impactación de segundos molares mandibulares (Tabla II) (Evans; Cho et al.; Shapira et al.; Sonis \& Ackerman; Fu et al.; Cassetta et al.).
Se analizó el lado afectado (segundo molar mandibular impactado) como grupo de estudio (GE) y el lado contra-lateral (segundo molar mandibular no impactado) como grupo control (GC), para realizar una comparación del comportamiento de las diferentes medidas angulares (Fig. 3).

Análisis estadístico: Las mediciones recolectadas de 
RAMíREZ, O. D.; UPEGUI, Z. J. C.; ESPINAL, B. G. E. \& CHAURRA, M. R. C. Caracterización de la impactación de segundos molares mandibulares permanentes en una población sudamericana. Int. J. Odontostomat., 11(2):165-172, 2017.

Tabla I. Concordancia intraobservador mediante el CCl.

\begin{tabular}{|c|c|c|c|c|}
\hline \multirow{2}{*}{ Grupo } & \multirow{2}{*}{ Evaluador 1} & \multirow{2}{*}{$\mathrm{CCl}$} & \multicolumn{2}{|c|}{ Intervalo de confianza 95\% } \\
\hline & & & L inferior & L superior \\
\hline \multirow{3}{*}{ Estudio } & AIM & 0,974 & 0,923 & 0,991 \\
\hline & APO & 0,959 & 0,882 & 0,986 \\
\hline & AIP & 0,966 & 0,902 & 0,988 \\
\hline \multirow{3}{*}{ Control } & AIM & 0,938 & 0,827 & 0,979 \\
\hline & APO & 0,864 & 0,643 & 0,952 \\
\hline & AIP & $\begin{array}{r}0,913 \\
\text {. }\end{array}$ & 0,760 & $\begin{array}{l}\text { 0,970 } \\
\text { IP. Áno }\end{array}$ \\
\hline
\end{tabular}

Tabla II. Medidas angulares usadas para evaluar la impactación del segundo molar mandibular.

\section{ÁNGULO DEFINICIÓN OPERACIONAL EJES / PLANOS}

\section{Ángulo intermolar (AIM)}

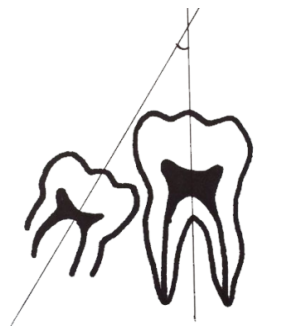

Ángulo del plano oclusal

(APO)

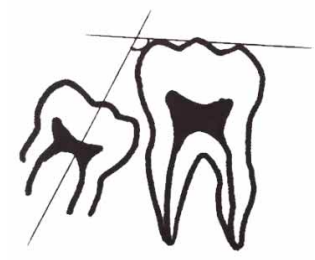

\section{Ángulo oclusal interplanar}

(AIP)

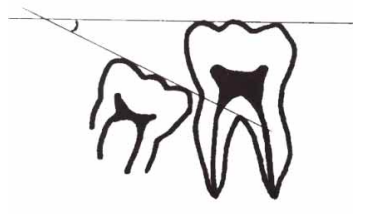

Intersección de los dos ejes Eje Iongitudinal: línea que longitudinales del primer y bisecta el máximo ancho segundo molar mandibular mesiodistal del diente y la permanente (ángulo interno). furcación de la raíz.

Intersección entre el plano Plano oclusal: unión del os oclusal y el eje longitudinal del dos puntos más superiores de segundo molar mandibular las cúspides mesial y distal del (ángulo interno). primer molar.

Intersección entre los planos oclusales del primer y segundo molar mandibular permanente (ángulo interno).
Plano oclusal: unión del os dos puntos más superiores de las cúspides mesial y distal del primer molar y del segundo molar. 


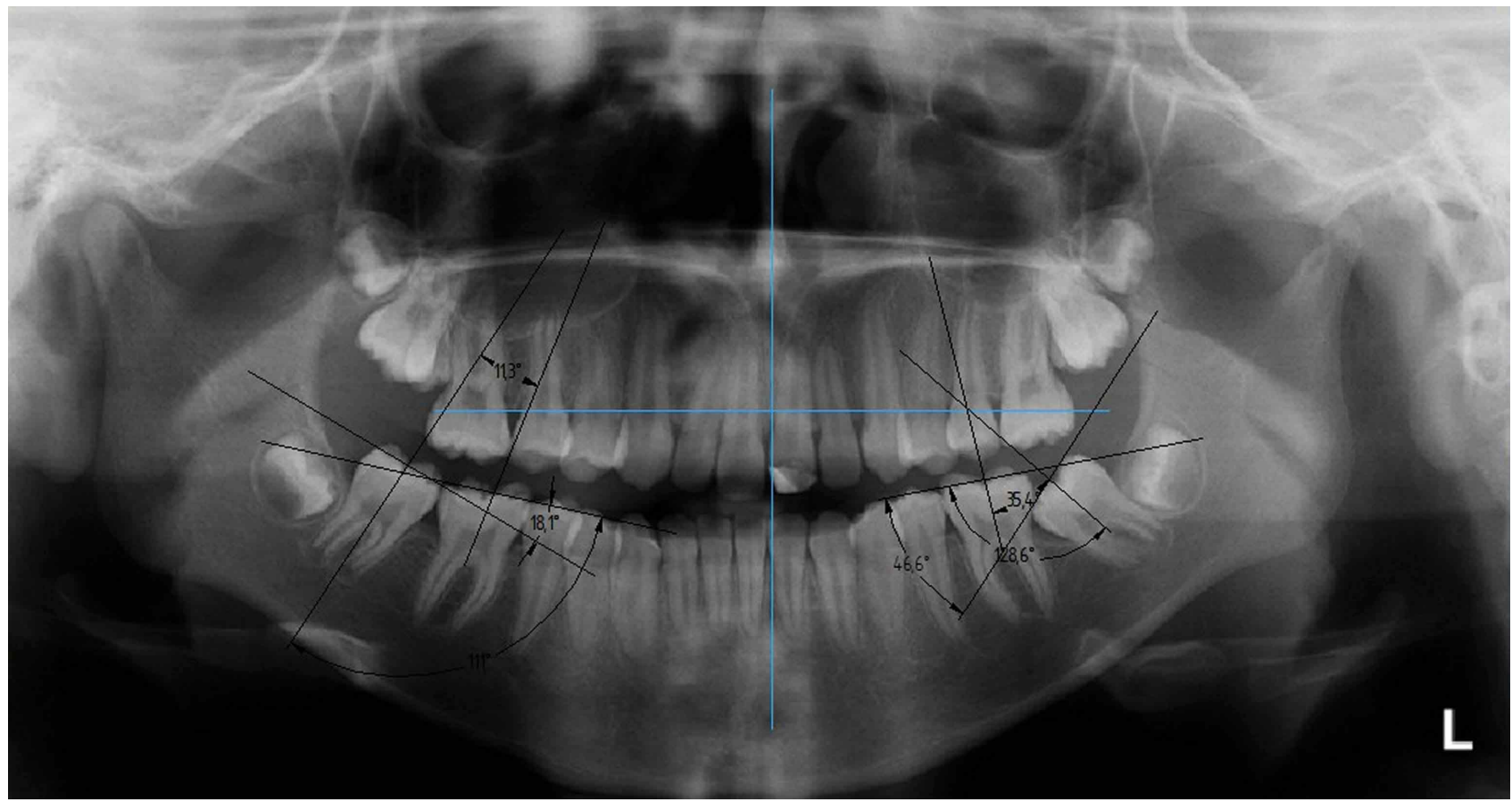

Fig. 3. Muestra de trazado de radiografía panorámica

las variables de este estudio se digitalizaron en una base de datos y hoja de cálculo en Excel. Se utilizó el paquete estadístico SPSS versión 21.0 (IBM) para el análisis final de los datos. Para las características de los pacientes se calcularon frecuencias relativas y absolutas; para la descripción de los ángulos se usaron medianas percentil 25 y 75 . Para definir si había diferencias entre el lado impactado y no impactado se utilizó la prueba de Wilcoxon; y para comparar los resultados según sexo se utilizó la prueba de $U$ de Mann Whitney.

Aspectos éticos: El estudio cumplió con los requisitos para investigación en salud según la normativa internacional y nacional (Ministerio de Salud y Protección Social, 1993; World Medical Association General Assembly, 2004). Se utilizó una fuente secundaria de datos, y se garantizaron la confidencialidad y el anonimato de los pacientes registrados en las historias clínicas. Se contó con la aprobación del Comité de Bioética de la Facultad de Odontología de la Universidad de Antioquia (Concepto 10-2015 del Acta 03 de junio 5 de 2015).

\section{RESULTADOS}

En el estudio se evaluaron un total de 28 radiografías, en cada una se realizó medición tanto del lado impactado como del no impactado. La prevalencia fue de 3,5 por cada 1000 radiografías revisadas. El grupo de estudio estuvo igualmente distribuido según sexo, la edad osciló entre 11 y 21 años, el 50 $\%$ tenían 12 años o menos con una variabilidad entre 11-16 años; el lado más impactado fue el derecho $(60,7 \%)$.

Al comparar el lado impactado con el contralateral no impactado, se observó que el primero tuvo ángulos más altos en las tres medidas tomadas; la diferencia entre ambos grupos para las tres mediciones fue estadísticamente significativa (Tabla III).

Los ángulos intermolar (AIM) e interplanar (AIP) fueron los que arrojaron las diferencias más grandes. Para el AIM el $50 \%$ de las mediciones el ángulo fue de $41^{\circ}$ o menos para el GE, mientras que para el GC fue de $4,7^{\circ}$ o menos. Para el APO el $50 \%$ del GE tuvo un ángulo de $136,8^{\circ}$ o menos y el GC $99,6^{\circ}$ o menos. Para el AIP el $50 \%$ del GE tuvo un ángulo de $43,9^{\circ}$ o menos y el GC $5,5^{\circ}$ o menos (Tabla III).

Al comparar los diferentes ángulos entre hombres y mujeres no se encontraron diferencias estadísticamente significativas, los valores fueron similares entre ellos (Tabla IV). 
Tabla III. Características de los ángulos.

\begin{tabular}{lccr}
\hline Ángulo & Estudio Me (Q1 -Q3) & Control Me (Q1 -Q3) & Valor $\mathbf{p}$ \\
\hline AIM & $41,0(31,1-52,9)$ & $4,7(2,0-6,4)$ & 0,0001 \\
APO & $136,8(126,5-144,8)$ & $99,6(95,9-104,8)$ & 0,0001 \\
AIP & $43,9(33,2-52,2)$ & $5,5(1,8-7,9)$ & 0,0001 \\
\hline
\end{tabular}

Me: Mediana - Q1: Percentil 25, Q3: Percentil 75

AIM: Ángulo intermolar - APO: Ángulo del plano oclusal - AIP: Ángulo del plano oclusal interplanar

* Valor $p$ de la prueba de Wilcoxon

Tabla IV. Características de los ángulos según sexo.

\begin{tabular}{lcccc}
\hline \multicolumn{1}{c}{ Angulo } & & Hombre Me (Q1 -Q3) & Mujer Me (Q1 -Q3) & Valor $\mathbf{p}^{\text {* }}$ \\
\hline \multirow{2}{*}{ rupo de } & AIM & $39,1(28,2-60,8)$ & $41,0(33,0-45,6)$ & 0,839 \\
sstudio & APO & $139,0(125,5-152,7)$ & $136,1(127,4-138,9)$ & 0,635 \\
& AIP & $44,6(32,9-60,6)$ & $41,3(33,4-51,4)$ & 0,769 \\
& AIM & $3,8(1,2-5,7)$ & $4,8(2,5-10,3)$ & 0,401 \\
po control & APO & $97,6(95,0-101,9)$ & $100,2(98,1-106,9)$ & 0,285 \\
& AIP & $6,0(1,8-7,8)$ & $5,0(1,8-7,9)$ & 0,910 \\
\hline
\end{tabular}

\section{DISCUSIÓN}

La prevalencia de la impactación de segundos molares mandibulares ha sido objeto de estudio por varios autores; para poblaciones europeas, se reportan prevalencias del 0,15\% (Varpio \& Wellfelt), 0,2 \% (Bondemark \& Tsiopa) y del 1,36 \% (Cassetta et al.); en asiáticos, hay un reporte del $0,65 \%$ en población Taiwanesa ( $\mathrm{Fu}$ et al.) y del 1,4\% en israelíes (Shapira et al.); en americanos, se encontró una prevalencia del $2,3 \%$ en una población chino-americana (Shapira et al.). No se encontró información disponible para poblaciones sudamericanas. Este estudio arrojó una prevalencia 0,35\% para impactaciones de tipo unilateral, después de analizar las radiografías de 6785 pacientes durante un período de año y medio, es decir, la tendencia es similar a la de muestras étnicas europeas. Aunque la muestra final fue pequeña, los valores de prevalencia arrojados están acordes con los reportes de la literatura.

Para este estudio, tanto hombres como mujeres presentaron la misma proporción de impactación, presentándose con mayor frecuencia en el lado derecho. Este resultado está de acuerdo a lo encontrado por Cassetta et al., Varpio \& Wellfelt y Fu et al., que aunque describen más alteraciones de la erup- ción del segundo molar mandibular en hombres que en mujeres, observaron un predominio de impactación en el lado derecho. Contrariamente, Cho et al. mostraron una mayor frecuencia de casos de manera unilateral en el lado izquierdo. Con respecto al sexo, otro estudio manifestó no haber encontrado diferencias (Bondemark \& Tsiopa), mientras que autores como Baccetti (2000) indican que sí las hay, pero muy pequeñas. Por lo tanto, no se puede hacer ninguna conclusión en cuanto a la existencia de una correlación entre el sexo, lado afectado y la impactación de segundos molares mandibulares.

Si bien es cierto que es difícil predecir con certeza lo biológico, al hacer un análisis geométrico de la relación que existe entre el cuerpo mandibular $u$ otras estructuras anatómicas con relación a los segundos molares inferiores en radiografías panorámicas, sería posible conocer el riesgo de impactación que puedan llegar a presentar, y utilizar las angulaciones estudiadas como una medida preventiva a edades tempranas, ya que se ha comprobado que la inclinación axial del segundo molar mandibular se mantiene casi invariable desde que la corona se ha calcificado (estadio de Nolla 6 ) hasta que la formación radicular se encuentra completa (Tsai, 2000). 
En este estudio se utilizaron tres angulaciones reportadas en la literatura para evaluar la inclinación del segundo molar mandibular y su posible riesgo de impactación en panorámicas: el ángulo intermolar (Evans; Cho et al.; Sonis \& Ackerman), el interplanar (Fu et al.; Cassetta et al.) y el del plano oclusal (Shapira et al.). Asimismo, se decidió incluir sólo pacientes con impactación unilateral para poder comparar con mayor precisión la angulación mesiodistal entre ambos lados (GE y GC) en el mismo paciente. Los ángulos de impactación obtenidos que presentaron diferencias más marcadas entre el grupo estudio y el grupo control fueron los ángulos intermolar e interplanar. El rango del AIM estuvo entre $31,1^{\circ}$ y $52,9^{\circ}$, presentando gran diferencia con los resultados de Evans $\left(15^{\circ}-65^{\circ}\right)$, Cho et al. $\left(13^{\circ}-75^{\circ}\right)$ y Sonis \& Ackerman $\left(19^{\circ}-33^{\circ}\right)$, la cual puede estar relacionada con otras variables que no fueron analizadas en el presente estudio, tales como el patrón facial, la relación esquelética, presencia de apiñamiento, pérdida temprana de dientes deciduos, ausencia de primer y/o tercer molar permanente, y otras alteraciones dentales como supernumerarios, taurodontismo, dens evaginatus, y dens invaginatus. Para los otros dos ángulos, los rangos se correlacionaron con los de otros estudios (Shapira et al.; Fu et al., 2012; Cassetta et al.).

Podría inferirse que encontrar una inclinación del segundo molar mandibular mayor a $6,4^{\circ}$ para el AIM, $104,8^{\circ}$ para el APO, y de $7,9^{\circ}$ para el AIP, es un indicativo de que hay un riesgo alto de que este diente se impacte contra el primer molar, análisis preventivo que podría realizarse de manera fácil, rápida y económica desde edades tempranas. Se prefiere el uso del AIP por ser el que presenta diferencias más marcadas entre el GE y GC, y el más similar con respecto a los hallazgos encontrados en otros estudios.

Como autores, sugerimos analizar una posible asociación entre las angulaciones y otras variables clínicas y cefalométricas que puedan favorecer la impactación de segundos molares, como el apiñamiento posterior y las relaciones esqueléticas del paciente y también realizar estudios a largo plazo que permitan hacer un seguimiento del patrón de erupción del segundo molar mandibular.

AGRADECIMIENTOS. Al Centro de Investigaciones de la Facultad de Odontología de la Universidad de Antioquia, por su contribución en el desarrollo de las diferentes fases del proyecto. A Lucelly López López, Profesional en Sistemas de Información en Salud (Uni- versidad de Antioquia) y Especialista en Estadística (Universidad Nacional), por su valiosa colaboración en la organización de los datos y en el enfoque de la unidad de análisis. Y a todas aquellas personas que de alguna u otra manera permitieron el desarrollo de este proyecto.

RAMÍREZ, O. D.; UPEGUI, Z. J. C.; ESPINAL, B. G. E. \& CHAURRA, M. R. C. Characterization of Permanent mandibular second molars impaction in a southamerican population. Int. J. Odontostomat., 11(2):165-172, 2017.

ABSTRACT: The objective of this study was to describe the epidemiological characteristics of unilateral permanent mandibular second molars impacted, in a sample from Medellín, Colombia. It was a retrospective study in which we evaluated panoramic radiographs of 6785 patients, for a total of 28 radiographs analyzed in the final sample. The mandibular second molar impacted (study group) and the contralateral not impacted (control group) were correlated, with respect to three reference angles (intermolar, of the oclussal plane and interplanar), sex and impaction side. We found a prevalence of 3.5 for every 1000 radiographs evaluated. The most frequent side of impaction was the right side; two of the three angular measurements (intermolar and interplanar) had the biggest differences between the study and control group. We did not find significant statistical differences between males and females. It was concluded that the prevalence of impacted permanent mandibular second molars is low, there is a highest impaction in the right side, there are no differences between genders, and the use of the interplanar angle is preferable as a measurement to predict the impaction of permanent mandibular second molars $\left(>7.9^{\circ}\right)$.

KEY WORDS: impacted tooth, molar, panoramic $\mathrm{X}$-ray, epidemiology.

\section{REFERENCIAS BIBLIOGRÁFICAS}

Andreasen, J. O.; Petersen, J. \& Laskin, M. Textbook and Color Atlas of Tooth Impactions. St. Louis, Mosby, 1997.

Baccetti, T. Tooth anomalies associated with failure of eruption of first and second permanent molars. Am. J. Orthod. Dentofacial Orthop., 118(6):608-10, 2000.

Bondemark, L. \& Tsiopa, J. Prevalence of ectopic eruption, impaction, retention and agenesis of the permanent second molar. Angle Orthod., 77(5):773-8, 2007.

Cassetta, M.; Altieri, F.; Di Mambro, A.; Galluccio, G. \& Barbato, E. Impaction of permanent mandibular second molar: a retrospective study. Med. Oral Patol. Oral Cir. Bucal, 18(4):e564-8, 2013.

Cho, S. Y.; Ki, Y.; Chu, V. \& Chan, J. Impaction of permanent mandibular second molars in ethnic Chinese schoolchildren. J. Can. Dent. Assoc., 74(6):521, 2008.

Evans, R. Incidence of lower second permanent molar impaction. Br. J. Orthod., 15(3):199-203, 1988. 
RAMÍREZ, O. D.; UPEGUI, Z. J. C.; ESPINAL, B. G. E. \& CHAURRA, M. R. C. Caracterización de la impactación de segundos molares mandibulares permanentes en una población sudamericana. Int. J. Odontostomat., 11(2):165-172, 2017.

Fardi, A.; Kondylidou-Sidira, A.; Bachour, Z.; Parisis, N. \& Tsirlis, A. Incidence of impacted and supernumerary teeth-a radiographic study in a North Greek population. Med. Oral Patol. Oral Cir. Bucal., 16(1):e56-61, 2011.

Frazier-Bowers, S. A.; Puranik, C. P. \& Mahaney, M. C. The etiology of eruption disorders - further evidence of a 'genetic paradigm'. Semin. Orthod., 16(3):180-5, 2010.

Frykholm, A.; Malmgren, O.; Sämfors, K. A. \& Welander, U. Angular measurements in orthopantomography. Dentomaxillofac. Radiol., 6(2):77-81, 1977.

Fu, P. S.; Wang, J. C.; Wu, Y. M.; Huang, T. K.; Chen, W. C.; Tseng, Y. C.; Tseng, C. H. \& Hung, C. C. Impacted mandibular second molars. Angle Orthod., 82(4):670-5, 2012.

Kokich, V. G. \& Mathews, D. P. Surgical and orthodontic management of impacted teeth. Dent. Clin. North Am., 37(2):181-204, 1993

Kurol, J. Impacted and ankylosed teeth: why, when, and how to intervene. Am. J. Orthod. Dentofacial. Orthop., 129/4 Suppl.):S86-90, 2006.

Lindauer, S. J.; Rubenstein, L. K.; Hang, W. M.; Andersen, W. C. \& Isaacson, R. J. Canine impaction identified early with panoramic radiographs. J. Am. Dent. Assoc., 123(3):91-2, 1992.

Magnusson, C. \& Kjellberg, H. Impaction and retention of second molars: diagnosis, treatment and outcome. A retrospective followup study. Angle Orthod., 79(3):422-7, 2009.

Ministerio de Salud y Protección Social. Resolución No. 008430 de 1993 (4 de octubre de 1993). Por la cual se establecen las Normas Científicas, Técnicas y Administrativas para la Investigación en Salud. Bogotá, Ministerio de Salud y Protección Social, República de Colombia, 1993. Disponible en: https:// www.unisabana.edu.co/fileadmin/Documentos/Investigacion/ comite_de_etica/Res 8430_1993_-_Salud.pdf

Miyahira, Y. I.; Maltagliati, L. A.; Siqueira, D. F. \& Romano, R. Miniplates as skeletal anchorage for treating mandibular second molar impactions. Am. J. Orthod. Dentofacial Orthop., 134(1):145-8, 2008.

Power, S. M. \& Short, M. B. An investigation into the response of palatally displaced canines to the removal of deciduous canines and an assessment of factors contributing to favourable eruption. Br. J. Orthod., 20(3):215-23, 1993.

Raghoebar, G. M.; Boering, G.; Vissink, A. \& Stegenga, B. Eruption disturbances of permanent molars: a review. J. Oral. Pathol. Med., 20(4):159-66, 1991.

Shapira, Y.; Borell, G.; Nahlieli, O. \& Kuftinec, M. M. Uprighting mesially impacted mandibular permanent second molars. Angle Orthod., 68(2):173-8, 1998.

Shapira, Y.; Finkelstein, T.; Shpack, N.; Lai, Y. H.; Kuftinec, M. M. \& Vardimon, A. Mandibular second molar impaction. Part I: Genetic traits and characteristics. Am. J. Orthod. Dentofacial Orthop., 140(1):32-7, 2011.

Shokeir, M. H. Complete failure of eruption of all permanent teeth: an autosomal dominant disorder. Clin. Genet., 5(4):322-6, 1974.

Sonis, A. \& Ackerman, M. E-space preservation. Angle Orthod., 81(6):1045-9, 2011.

Stramotas, S.; Geenty, J. P.; Darendeliler, M. A.; Byloff, F.; Berger, J. \& Petocz, P. The reliability of crown-root ratio, linear and angular measurements on panoramic radiographs. Clin. Orthod. Res., 3(4):182-91, 2000.

Suri, L.; Gagari, E. \& Vastardis, H. Delayed tooth eruption: pathogenesis, diagnosis, and treatment. A literature review. Am. J. Orthod. Dentofacial Orthop., 126(4):432-45, 2004.

Tsai, H. H. Eruption process of the second molar. A. S. D. C. J. Dent. Child., 67(4):275-81, 2000.

Varpio, M. \& Wellfelt, B. Disturbed eruption of the lower second molar: clinical appearance, prevalence, and etiology. A. S. D. C. J. Dent. Child., 55(2):114-8, 1988.
Vedtofte, H.; Andreasen, J. O. \& Kjaer, I. Arrested eruption of the permanent lower second molar. Eur. J. Orthod., 21(1):31-40, 1999.

Warford, J. H. Jr.; Grandhi, R. K. \& Tira, D. E. Prediction of maxillary canine impaction using sectors and angular measurement. Am. J. Orthod. Dentofacial Orthop., 124(6):651-5, 2003.

Wellfelt, B. \& Varpio, M. Disturbed eruption of the permanent lower second molar: treatment and results. A. S. D. C. J. Dent. Child., 55(3):183-9, 1988

World Medical Association General Assembly. World Medical Association Declaration of Helsinki: ethical principles for medical research involving human subjects. J. Int. Bioethique, 15(1):1249, 2004.

Dirección para correspondencia:

Diana Ramírez Ossa

Facultad de Odontología

Universidad de Antioquia

Calle 64 \# 52-59

Medellín

COLOMBIA

E-mail: milena.ramirez@udea.edu.co

Recibido : 18-10-2016

Aceptado: 07-04-2017 